\title{
二元三次样条空间 $S_{3}^{1,2}\left(\Delta_{m n}^{(2)}\right)$ 的样条拟插值
}

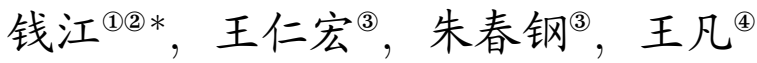 \\ (1) 河海大学理学院, 南京 210098 ; \\ (2) 河海大学水文水资源与水利工程科学国家重点实验室, 南京 210098; \\ (3) 大连理工大学数学科学学院, 大连 116024 ; \\ (4) 南京农业大学工学院基础课部, 南京 210031 \\ E-mail: qianjianghhu@sina.com, renhong@dlut.edu.cn, cgzhu@dlut.edu.cn, wangfan@njau.edu.cn
}

收稿日期: 2013-09-17; 接受日期: 2013-12-24; * 通信作者

国家自然科学基金 (批准号: 11271060 和 11290143)、河海大学博士后科研基金 (批准号: 2016-412051)、民用飞机专项 (批准号: MJ-F-2012-04) 和中央基本科研业务费 (批准号: DUT14YQ111) 资助项目

摘要 本文首先利用由两组具有局部最小支集的样条所组成的基函数, 构造非均匀 2 型三角剖分上二 元三次样条空间 $S_{3}^{1,2}\left(\Delta_{m n}^{(2)}\right)$ 的若干样条拟插值算子. 这些变差缩减算子由样条函数 $B_{i j}^{1}$ 支集上 5 个 网格点或中心和样条函数 $B_{i j}^{2}$ 支集上 5 个网格点处函数值定义. 这些样条拟插值算子具有较好的逼近 性, 甚至算子 $V_{m n}(f)$ 能保持近最优的三次多项式性. 然后利用连续模, 分析样条拟插值算子 $V_{m n}(f)$ 一致逼近于充分光滑的实函数. 最后推导误差估计.

关键词 二元样条 光滑余因子协调法 非均匀 2 型三角剖分 拟插值 连续模

MSC (2010) 主题分类 65D07, 41A25

\section{1 引言}

众所周知, 基于代数多项式形式的非均匀有理 B 样条 (NURBS) 是计算机辅助几何设计 (CAGD) 中目前最经典的曲线曲面模型, 它之所以成为现行的几何造型系统的标准, 主要在于它可以表示自由 曲线曲面和一些传统的解析几何模型, 如圆雉曲线等 ${ }^{[1-4]}$. 近年来, 为了保持 NURBS 模型良好的几何 性质, 如正性、单调性和凸性等, 克服其局限性如有理形式不便求导等, 人们提出并研究了一些基于代 数多项式和非多项式混合空间上的曲线曲面模型 ${ }^{[5-11]}$, 并且研究表明, 这些基于混合基底的样条极限 形式为 B 样条. 然而, 上述诸多几何造型方法共同的美中不足之处在于, 张量积形式的曲面次数往往 较高, 因为其次数是两参数方向的次数之和. 例如, 双三次 B 样条曲面的次数为 6 . 由于次数高, 曲面 上可能出现一些奇异点. 更进一步, 此类二元函数局限于矩形域上, 而且不具有保次数接近最优的多 项式性. 而基于光滑余因子协调法的多元样条可以避免这些不足. 因此, 本文将利用光滑余因子协调 法继续研究二元三次样条空间中的样条拟插值.

事实上, 光滑余因子协调法是确定多元样条维数 ${ }^{[12-16]}$ 和计算多元样条空间基函数 ${ }^{[17-20]}$ 的有效 工具, 这些问题研究往往很复杂但很重要, 而当前更深入的前沿研究主要是分片代数簇问题等 ${ }^{[21-25]}$. 有关样条空间 $S_{k}^{\mu}(\Delta)$ (即区域 $D$ 上关于剖分 $\Delta$ 具有 $\mu$ 阶连续偏导数的分片 $k$ 次多项式空间) 维数的 重要理论成果可参见文献 $[12,13,26]$. 近年来, 均匀 2 型三角剖分上的二元三次和四次样条空间的样 
条基函数已经被算出 ${ }^{[17,18]}$, 而且相应的样条拟插值算子也被构造出. 这些样条拟插值分别具有保次数 接近最优的多项式性. 非均匀 2 型三角剖分上的二元二次样条空间的样条拟插值算子在文献 $[27,28]$ 中给出. 由此, 基于数目确定的三角网点的离散线性泛函所定义的样条拟插值得以深入研究, 这些拟插

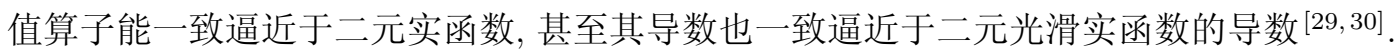

然而需要指出的是, 对于非均匀 2 型三角剖分情形, 由于缺少计算出样条基函数, 二元样条拟插 值的研究仅局限于二元二次样条情形. 由于不规则区域 (如含 “洞” 区域) 上曲面的构造需要非均匀剖 分下样条基函数, 因此, 非均匀剖分下多元样条逼近有时比均匀情形下重要. 利用光滑余因子协调法

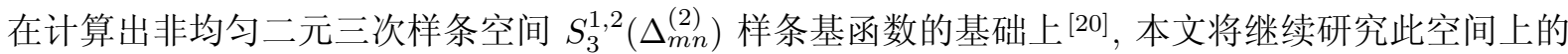
样条拟插值, 并且分析其一致逼近. 更进一步有关样条拟插值导数一致逼近充分光滑实函数导数的问 题将在其他论文中研究 ${ }^{[31]}$.

本文主要结构如下: 第 2 节首先回顾非均匀三次样条空间维数和样条基函数. 第 3 节构造由离散 线性泛函定义的三次样条拟插值算子, 这些线性泛函基于数目确定的三角网点. 诸多样条拟插值中所 谓最优样条拟插值能再生多项式空间 $\mathbb{P}_{2} \cup\left\{x^{2} y, x y^{2}\right\}$, 即保次数接近最优的三次多项式性. 第 4 节利 用连续模, 计算所谓最优样条拟插值算子一致逼近实函数的误差估计.

\section{2 样条空间 $S_{3}^{1,2}\left(\Delta_{m n}^{(2)}\right)$ 维数和样条基函数}

我们将区域 $\Omega=[a, b] \times[c, d]$ 剖分成 $m n$ 个小矩形区域 $\Omega_{i j}=\left[x_{i}, x_{i+1}\right] \times\left[y_{j}, y_{j+1}\right], i=0, \ldots, m-1$ 且 $j=0, \ldots, n-1$, 其中 $m$ 和 $n$ 是正数, 且 $a=x_{0}<x_{1}<\cdots<x_{m}=b, c=y_{0}<y_{1}<\cdots<y_{n}=d$. 这样通过连接每个矩形域 $\Omega_{i j}$ 中两条对角线, 我们得到一个 2 型三角剖分 $\Delta_{m n}^{(2)}$. 令 $h_{i}=x_{i}-x_{i-1}$, $k_{j}=y_{j}-y_{j-1}$. 特别地, 称三角剖分是均匀的, 若 $h_{i}=h_{i-1}$ 且 $k_{j}=k_{j-1}$; 否则称三角剖分是非均匀的.

二元三次分片多项式 $s(x, y) \in S_{3}^{1,2}\left(\Delta_{m n}^{(2)}\right)$ 称为非均匀二元样条函数, 若满足下述两条件:

(1) $s(x, y)$ 于水平与垂直网格线 $x=x_{i}, y=y_{j}$, 满足 $C^{1}$ 连续, 其中 $i=0, \ldots, m$, 且 $j=0, \ldots, n$;

(2) $s(x, y)$ 于每个胞腔对角线

$$
y-y_{j}-\frac{k_{j+1}}{h_{i+1}}\left(x-x_{i}\right)=0, \quad y-y_{j}+\frac{k_{j+1}}{h_{i+1}}\left(x-x_{i+1}\right)=0,
$$

满足 $C^{2}$ 连续, 其中 $i=0, \ldots, m-1$, 且 $j=0, \ldots, n-1$.

我们已经证明非均匀二元三次样条空间 $S_{3}^{1,2}\left(\Delta_{m n}^{(2)}\right)$ 维数与均匀情形下相同 ${ }^{[20]}$, 而均匀情形下空 间维数早已算出 ${ }^{[17]}$, 即

$$
\operatorname{dim} S_{3}^{1,2}\left(\Delta_{m n}^{(2)}\right)=2 m n+3 m+3 n+4 .
$$

而且, 我们也计算出非均匀样条空间 $S_{3}^{1,2}\left(\Delta_{m n}^{(2)}\right)$ 样条基函数表达式, 其由具有不同最小支集的两组样 条所组成, 两种最小支集分别如图 1 和 2 所示. 记两种支集上的每个三角形胞腔分别为 $\Delta_{u}^{1}$ 和 $\Delta_{v}^{2}$, 定义其上的样条函数分别为 $B_{i j}^{1}\left(\Delta_{u}^{1}\right)(u=1,2, \ldots, 16)$ 和 $B_{i j}^{2}\left(\Delta_{v}^{2}\right)(v=1,2, \ldots, 24)$.

样条函数 $B_{i j}^{1}(x, y)$ 的支集以点 $\left(x_{i}, y_{j}\right)$ 为中心, 以点

$$
\begin{array}{lll}
P_{1}\left(x_{i-1}, y_{j+1}\right), & P_{2}\left(x_{i-1}, y_{j}\right), & P_{3}\left(x_{i-1}, y_{j-1}\right), \\
P_{4}\left(x_{i}, y_{j-1}\right), \quad P_{5}\left(x_{i+1}, y_{j-1}\right), & P_{6}\left(x_{i+1}, y_{j}\right), \\
P_{7}\left(x_{i+1}, y_{j+1}\right), & P_{8}\left(x_{i}, y_{j+1}\right), & P_{9}\left(x_{i}, y_{j}\right)
\end{array}
$$

为顶点, 如图 1 所示. 
样条函数 $B_{i j}^{2}(x, y)$ 的支集以点 $\left(\frac{x_{i}+x_{i+1}}{2}, \frac{y_{j}+y_{j+1}}{2}\right)$ 为中心, 以点

$$
\begin{array}{lll}
Q_{1}\left(x_{i}, y_{j+2}\right), & Q_{2}\left(x_{i-1}, y_{j+1}\right), & Q_{3}\left(x_{i-1}, y_{j}\right), \\
Q_{4}\left(x_{i}, y_{j-1}\right), & Q_{5}\left(x_{i+1}, y_{j-1}\right), & Q_{6}\left(x_{i+2}, y_{j}\right), \\
Q_{7}\left(x_{i+2}, y_{j+1}\right), & Q_{8}\left(x_{i+1}, y_{j+2}\right), & Q_{9}\left(x_{i}, y_{j+1}\right), \\
Q_{10}\left(x_{i}, y_{j}\right), & Q_{11}\left(x_{i+1}, y_{j}\right), & Q_{12}\left(x_{i+1}, y_{j+1}\right)
\end{array}
$$

为顶点, 如图 2 所示.

通过平移样条基函数, 样条函数 $B_{i j}^{1}(x, y)$ 和 $B_{i j}^{2}(x, y)$ 在矩形域 $\Omega$ 上不全为 0 的指标集分别为

$$
\begin{aligned}
& I_{1}=\{(i, j)=(\alpha, \beta): 0 \leqslant \alpha \leqslant m, 0 \leqslant \beta \leqslant n\}, \\
& I_{2}=\{(i, j)=(\alpha, \beta):-1 \leqslant \alpha \leqslant m,-1 \leqslant \beta \leqslant n\},
\end{aligned}
$$

从而, 矩形域 $\Omega$ 上不全为 0 的线性无关的样条函数 $B_{i j}^{1}(x, y)$ 和 $B_{i j}^{2}(x, y)$ 总数为 $2 m n+3 m+3 n+5$. 因 此, 由 (2.1), 其比样条空间维数 $S_{3}^{1,2}\left(\Delta_{m n}^{(2)}\right)$ 多 1. 于是, 我们构造了非均匀二元三次样条空间 $S_{3}^{1,2}\left(\Delta_{m n}^{(2)}\right)$ 的样条基函数, 如下定理所述.

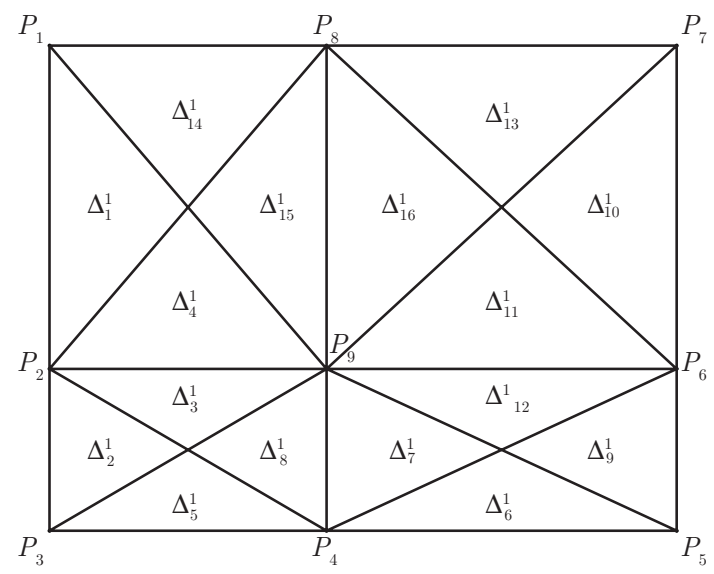

图 $1 B_{i j}^{1}(x, y)$ 最小支集中的 16 个三角形胞腔

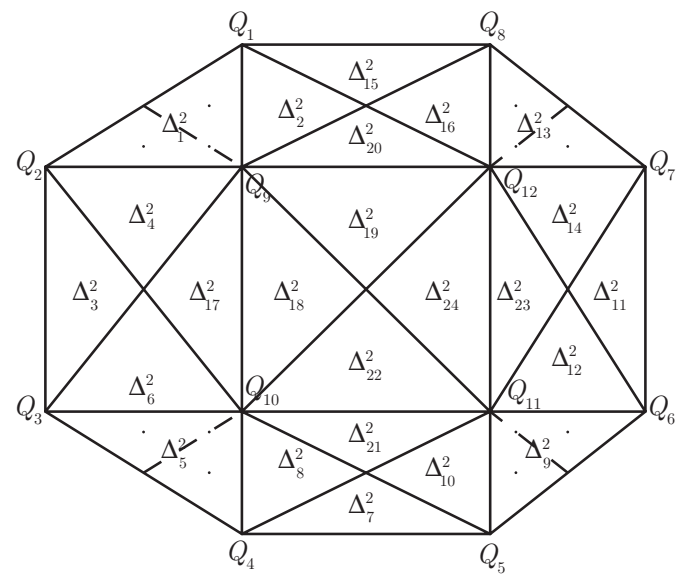

图 $2 B_{i j}^{2}(x, y)$ 最小支集中的 24 个三角形胞腔 
定理 $2.1^{[20]}$ 设任意 $\left(i_{0}, j_{0}\right) \in I_{1},\left(i_{1}, j_{1}\right) \in I_{2}$, 记

$$
\begin{aligned}
& \mathbb{B}^{1}=\left\{B_{i j}^{1}:(i, j) \in I_{1} \backslash\left\{\left(i_{0}, j_{0}\right)\right\}\right\} \cup\left\{B_{i j}^{2}:(i, j) \in I_{2}\right\}, \\
& \mathbb{B}^{2}=\left\{B_{i j}^{2}:(i, j) \in I_{2} \backslash\left\{\left(i_{1}, j_{1}\right)\right\}\right\} \cup\left\{B_{i j}^{1}:(i, j) \in I_{1}\right\},
\end{aligned}
$$

则非均匀三次样条空间 $S_{3}^{1,2}\left(\Delta_{m n}^{(2)}\right)$ 的基函数为 $\mathbb{B}^{1}$ 或 $\mathbb{B}^{2}$.

通过样条基函数于每个三角形胞腔内 10 点 (即各边三等分点和重心) 处的函数值平移, 我们得到 这些具有最小支集的样条函数满足下述性质.

定理 $2.2^{[20]}$ 对一切 $(x, y) \in \Omega$, 成立

$$
\begin{aligned}
& \sum_{(i, j) \in I_{1}} B_{i j}^{1}(x, y)=1+\lambda, \\
& \sum_{(i, j) \in I_{2}} B_{i j}^{2}(x, y)=-\lambda, \\
& \sum_{(i, j) \in I_{1}} B_{i j}^{1}(x, y)+\sum_{(i, j) \in I_{2}} B_{i j}^{2}(x, y)=1,
\end{aligned}
$$

其中 $\lambda \neq 0,-1$.

当 $\lambda \in(-1,0)$ 时, 样条函数 $B_{i j}^{1}(x, y), B_{i j}^{2}(x, y) \in[0,1)$. 特别地, 当 $\lambda=-0.3,-0.6$ 时, $B_{i j}^{1}(x, y)$ 和 $B_{i j}^{2}(x, y)$ 如图 3 所示.

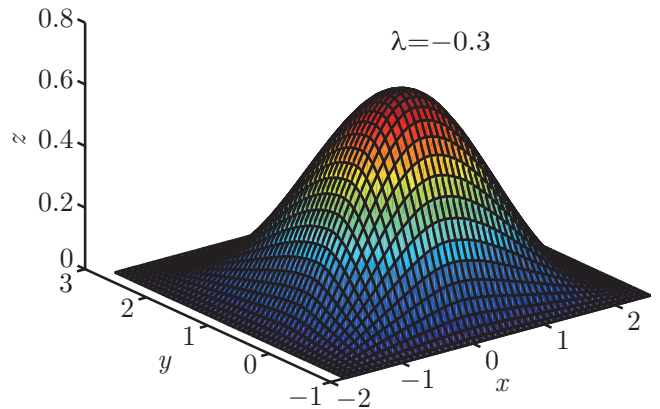

(a)

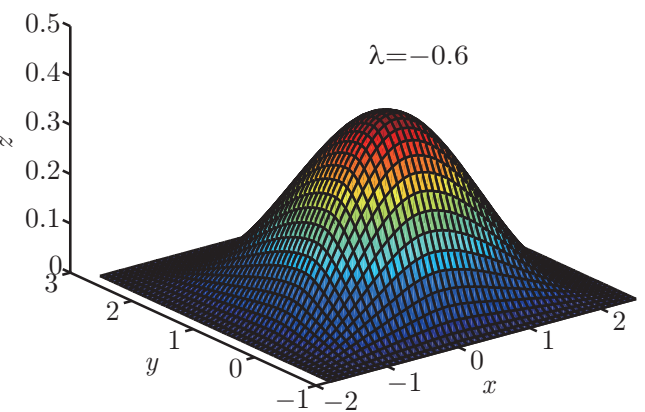

(c)

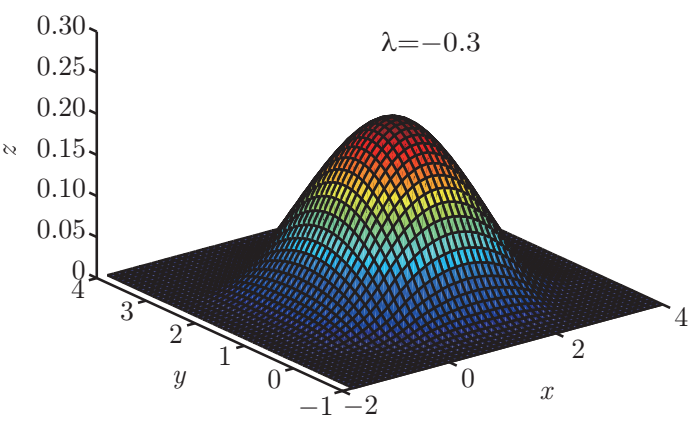

(b)

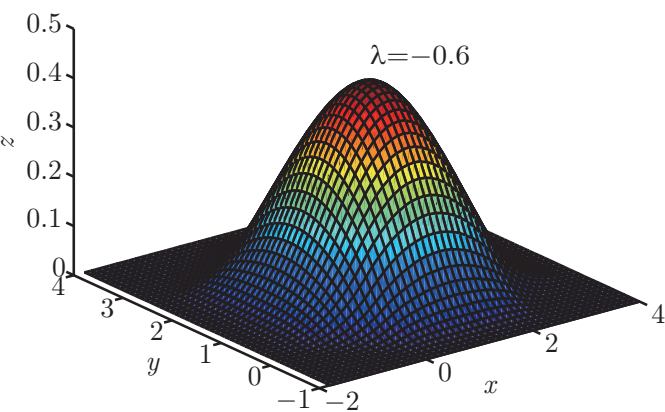

(d)

图 3 (a) $B_{i j}^{1}(x, y), \lambda=-0.3$; (b) $B_{i j}^{2}(x, y), \lambda=-0.3$; (c) $B_{i j}^{1}(x, y), \lambda=-0.6$; (d) $B_{i j}^{2}(x, y)$, $\lambda=-0.6$ 


\section{3 样条空间 $S_{3}^{1,2}\left(\Delta_{m n}^{(2)}\right)$ 的样条拟插值表达式}

本节将深入研究并构造由离散线性泛函定义的样条拟插值算子, 这些线性泛函基于上节两种最小 支集内的若干内网点. 这些样条拟插值算子具有保二元多项式性, 而且误差分析也将表明其中保最优 次数多项式性的变差缩减算子 $V_{m n}(f)$ 能一致逼近于充分光滑实函数.

记具有局部最小支集的两组样条函数分别为 $B_{i j}^{1}(x, y)$ 和 $B_{i j}^{2}(x, y)$, 其中 $\lambda \neq-1,0$. 定义线性算 子 $\tilde{V}_{m n}$ 如下:

$$
\begin{aligned}
\tilde{V}_{m n}: C(\Omega) \rightarrow S_{3}^{1,2}\left(\Delta_{m n}^{(2)}\right): & \\
\tilde{V}_{m n}(f) & =\sum_{(i, j) \in I_{1}} \tilde{\lambda}_{i j}(f) B_{i j}^{1}(x, y)+\sum_{(i, j) \in I_{2}} \tilde{\mu}_{i j}(f) B_{i j}^{2}(x, y),
\end{aligned}
$$

其中点 $\left(x_{i}, y_{j}\right)$ 和 $\left(\frac{x_{i}+x_{i+1}}{2}, \frac{y_{j}+y_{j+1}}{2}\right)$ 分别为 $B_{i j}^{1}(x, y)$ 和 $B_{i j}^{2}(x, y)$ 最小支集的中心, 且

$$
\begin{aligned}
\tilde{\lambda}_{i j}(f)= & \sigma_{1} f\left(x_{i}, y_{j}\right)+\alpha_{1} f\left(\frac{x_{i-1}+x_{i}}{2}, \frac{y_{j}+y_{j+1}}{2}\right)+\beta_{1} f\left(\frac{x_{i}+x_{i+1}}{2}, \frac{y_{j}+y_{j+1}}{2}\right) \\
& +\gamma_{1} f\left(\frac{x_{i-1}+x_{i+1}}{2}, \frac{y_{j-1}+y_{j}}{2}\right)+\delta_{1} f\left(\frac{x_{i}+x_{i+1}}{2}, \frac{y_{j-1}+y_{j}}{2}\right), \\
\tilde{\mu}_{i j}(f)= & \sigma_{2} f\left(\frac{x_{i}+x_{i+1}}{2}, \frac{y_{j}+y_{j+1}}{2}\right) \\
& +\alpha_{2} f\left(x_{i}, y_{j+1}\right)+\beta_{2} f\left(x_{i+1}, y_{j+1}\right)+\gamma_{2} f\left(x_{i}, y_{j}\right)+\delta_{2} f\left(x_{i+1}, y_{j}\right) .
\end{aligned}
$$

如上离散线性泛函 (3.2) 中系数 $\sigma_{i}, \alpha_{i}, \beta_{i}, \gamma_{i}$ 和 $\delta_{i}(i=1,2)$ 为实常数.

定理 3.1 若由 $C(\Omega)$ 映射到 $S_{3}^{1,2}\left(\Delta_{m n}^{(2)}\right)$ 的变差缩减算子 $V_{m n}^{1}(f)$ 设为

$$
V_{m n}^{1}(f)=\sum_{(i, j) \in I_{1}} \lambda_{i j}^{1}(f) B_{i j}^{1}(x, y)+\sum_{(i, j) \in I_{2}} \mu_{i j}^{1}(f) B_{i j}^{2}(x, y)
$$

其中

$$
\begin{aligned}
\lambda_{i j}^{1}(f)= & \frac{1}{3(1+\lambda)} f\left(x_{i}, y_{j}\right)+\gamma f\left(\frac{x_{i-1}+x_{i}}{2}, \frac{y_{j}+y_{j+1}}{2}\right)-\gamma f\left(\frac{x_{i}+x_{i+1}}{2}, \frac{y_{j}+y_{j+1}}{2}\right) \\
& -\gamma f\left(\frac{x_{i-1}+x_{i+1}}{2}, \frac{y_{j-1}+y_{j}}{2}\right)+\gamma f\left(\frac{x_{i}+x_{i+1}}{2}, \frac{y_{j-1}+y_{j}}{2}\right), \\
\mu_{i j}^{1}(f)= & -2\left(\frac{1}{3 \lambda}+\alpha+\beta\right) f\left(\frac{x_{i}+x_{i+1}}{2}, \frac{y_{j}+y_{j+1}}{2}\right) \\
& +\alpha f\left(x_{i}, y_{j+1}\right)+\beta f\left(x_{i+1}, y_{j+1}\right)+\beta f\left(x_{i}, y_{j}\right)+\alpha f\left(x_{i+1}, y_{j}\right),
\end{aligned}
$$

且 $\alpha, \beta$ 和 $\gamma$ 为实常数, 则对 $\forall(x, y) \in \Omega$, 成立

$$
V_{m n}^{1}(f) \equiv f(x, y), \quad \forall f(x, y) \in \mathbb{P}_{1} .
$$

证明 易知, 只要证明结论 (3.5) 对 $p(x, y)=1, x, y$ 精确成立即可, 也就是要证明结论 (3.5) 于矩 形域 $\Omega$ 上的每个三角形胞腔精确成立. 不失一般性, 我们考虑 $B_{i j}^{1}$ 的最小支集上胞腔 $\Delta_{11}^{1}$ 或 $B_{i j}^{2}$ 的 最小支集上胞腔 $\Delta_{22}^{2}$. 我们将 $B_{i j}^{1}$ 的最小支集上 4 个三角形胞腔与 $B_{i j}^{2}$ 的最小支集上 7 个三角形胞 腔分别平移到样条函数不恒为 0 的三角形胞腔 $\Delta_{11}^{1}$ 和 $\Delta_{22}^{2}$, 则得到样条拟插值表达式为

$$
V_{m n}^{1}(f)=\lambda_{i+1, j}^{1}(f) B_{i+1, j}^{1}+\lambda_{i, j}^{1}(f) B_{i, j}^{1}+\lambda_{i+1, j+1}^{1}(f) B_{i+1, j+1}^{1}+\lambda_{i, j+1}^{1}(f) B_{i, j+1}^{1}
$$




$$
\begin{aligned}
& +\mu_{i+1, j-1}^{1}(f) B_{i+1, j-1}^{2}+\mu_{i, j-1}^{1}(f) B_{i, j-1}^{2}+\mu_{i-1, j-1}^{1}(f) B_{i-1, j-1}^{2}+\mu_{i+1, j}^{1}(f) B_{i+1, j}^{2} \\
& +\mu_{i j}^{1}(f) B_{i, j}^{2}+\mu_{i-1, j}^{1}(f) B_{i-1, j}^{2}+\mu_{i, j+1}^{1}(f) B_{i, j+1}^{2} .
\end{aligned}
$$

通过上式, 容易证明结论 (3.5) 对 $f(x, y)=1$ 恒成立. 更进一步, 考虑到 (3.6) 中每个二元三次 样条函数由在相应支集每个三角形胞腔上的 10 个点处函数值唯一确定, 这 10 个点分别为 3 个顶点、 每条边上非顶点的 2 个三等分点和重心, 所以, 只需要证明 (3.6) 于此 10 点处对 $f(x, y)=x, y$ 精确 成立即可. 具体而言, 我们还需要计算出 $B_{i j}^{2}$ 支集中 $\Delta_{1}^{2}, \Delta_{13}^{2}, \Delta_{5}^{2}$ 和 $\Delta_{9}^{2}$ 每个三角形胞腔上其他三个 点处的函数值. 如图 2 所示, 我们用连接 1 个顶点与对边中点的虚线分别将此 4 个三角形胞腔每个分 成 2 个三角形胞腔, 并且注意到, 新产生的 2 个三角形胞腔上的样条函数表达式与原来一个胞腔上的 相同. 这样只需要计算出新得到的 8 个三角形胞腔的重心和虚线上靠近 $B_{i j}^{2}$ 支集内网点的三等分点的 函数值.

计算表明, 将 $B_{i j}^{1}$ 的支集上 4 个三角形胞腔和 $B_{i j}^{2}$ 的支集上 7 个三角形胞腔分别平移到样条函 数不恒为 0 的三角形胞腔 $\Delta_{11}^{1}$ 和 $\Delta_{22}^{2}$ 时, (3.6) 于 $\Delta_{11}^{1}$ 或 $\Delta_{22}^{2}$ 上 10 个点处对 $f(x, y)=x, y$ 精确成 立. 因此, 我们证明了所构造的样条拟插值算子 $V_{m n}^{1}(f)$ 能再生二元一次多项式空间.

更进一步, 有下面的定理.

定理 3.2 若由 $C(\Omega)$ 映射到 $S_{3}^{1,2}\left(\Delta_{m n}^{(2)}\right)$ 的变差缩减算子 $V_{m n}^{2}(f)$ 设为

$$
V_{m n}^{2}(f)=\sum_{(i, j) \in I_{1}} \lambda_{i j}^{2}(f) B_{i j}^{1}(x, y)+\sum_{(i, j) \in I_{2}} \mu_{i j}^{2}(f) B_{i j}^{2}(x, y)
$$

其中

$$
\begin{aligned}
\lambda_{i j}^{2}(f)= & \frac{1}{3(1+\lambda)} f\left(x_{i}, y_{j}\right) \\
\mu_{i j}^{2}(f)= & -2\left(\frac{1}{3 \lambda}+2 \alpha\right) f\left(\frac{x_{i}+x_{i+1}}{2}, \frac{y_{j}+y_{j+1}}{2}\right) \\
& +\alpha\left[f\left(x_{i}, y_{j+1}\right)+f\left(x_{i+1}, y_{j+1}\right)+f\left(x_{i}, y_{j}\right)+f\left(x_{i+1}, y_{j}\right)\right]
\end{aligned}
$$

且 $\alpha$ 为实常数, 则对 $\forall(x, y) \in \Omega$, 成立

$$
V_{m n}^{2}(f) \equiv f(x, y), \quad \forall f(x, y) \in \mathbb{P}_{1} \cup \operatorname{span}\{x y\} .
$$

类似定理 3.1 的证明, 我们只需要证明 (3.9) 于此 10 个点 (各边三等分点和重心) 处对 $f(x, y)=1$, $x, y, x y$ 精确成立即可.

最后, 我们构造能生成近最优次数 (即 3 次) 二元多项式的样条拟插值算子.

定理 3.3 若由 $C(\Omega)$ 映射到 $S_{3}^{1,2}\left(\Delta_{m n}^{(2)}\right)$ 的变差缩减算子 $V_{m n}(f)$ 设为

$$
V_{m n}(f)=\sum_{(i, j) \in I_{1}} \lambda_{i j}(f) B_{i j}^{1}(x, y)+\sum_{(i, j) \in I_{2}} \mu_{i j}(f) B_{i j}^{2}(x, y),
$$

其中

$$
\begin{aligned}
& \lambda_{i j}(f)=\frac{1}{3(1+\lambda)} f\left(x_{i}, y_{j}\right), \\
& \mu_{i j}(f)=-\frac{4}{3 \lambda} f\left(\frac{x_{i}+x_{i+1}}{2}, \frac{y_{j}+y_{j+1}}{2}\right)
\end{aligned}
$$




$$
+\frac{1}{6 \lambda}\left[f\left(x_{i}, y_{j+1}\right)+f\left(x_{i+1}, y_{j+1}\right)+f\left(x_{i}, y_{j}\right)+f\left(x_{i+1}, y_{j}\right)\right]
$$

则对 $\forall(x, y) \in \Omega$, 成立

$$
V_{m n}(f) \equiv f(x, y), \quad \forall f(x, y) \in \mathbb{P}_{2} \cup \operatorname{span}\left\{x^{2} y, x y^{2}\right\}
$$

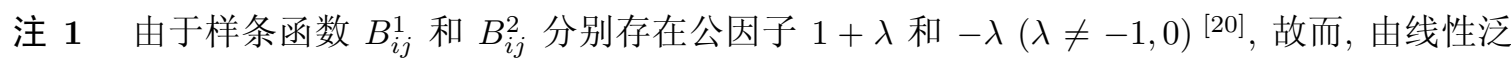
函 (3.11) 可知, 所谓最优样条拟插值算子 $V_{m n}(f)$ 与参数 $\lambda$ 无关.

注 2 注 1 表明, 样条拟插值算子 (3.10) 由基于 $B_{i j}^{1}$ 最小支集的中心和 $B_{i j}^{2}$ 最小支集的 5 个内 网点的线性泛函 (3.11) 所唯一确定. 因此, 不可能构造出由形如离散线性泛函 (3.2) 所定义的样条拟 插值算子再生多项式空间 $\operatorname{span}\left\{x^{3}, y^{3}\right\}$.

\section{4 样条拟插值算子 $V_{m n}(f)$ 逼近于二元实函数的误差估计}

由于具有不同支集的两组样条函数 $B_{i j}^{1}$ 和 $B_{i j}^{2}$ 能张成整个三次样条空间 $S_{3}^{1,2}\left(\Delta_{m n}^{2}\right)$, 而由 (3.10) 定义的样条拟插值算子能生成接近最优次数的多项式, 故而, 我们利用样条拟插值 $V_{m n}(f)$ 一致逼近 空间 $S_{3}^{1,2}\left(\Delta_{m n}^{2}\right)$, 并且分析其误差估计.

我们定义有序数对 $(x, y)$ 的 Euclid 范数为 $|(x-y)|=\sqrt{x^{2}+y^{2}}$, 且定义 $f(K)$ 的连续模如下:

$$
\omega_{K}(f, \delta)=\sup \{|f(x, y)-f(u, v)|:(x, y),(u, v) \in K,|(x, y)-(u, v)|<\delta\}
$$

其中 $K \in \mathbb{R}^{2}$ 是紧集.

设紧集 $K$ 是包含 $\Omega$ 的开集的闭包, 我们选择充分大的 $m$ 和 $n$, 使得两种支集的中心位于 $K$ 的 内部, 则定义样条函数 $B_{i j}^{1}$ 和 $B_{i j}^{2}$ 最小支集的最大半径分别为

$$
\begin{aligned}
& r_{m n, 1}=\max _{0 \leqslant i \leqslant m, 0 \leqslant j \leqslant n}\left\{\left|P_{9} P_{1}\right|,\left|P_{9} P_{3}\right|,\left|P_{9} P_{5}\right|,\left|P_{9} P_{7}\right|\right\}, \\
& r_{m n, 2}=\max _{-1 \leqslant i \leqslant m,-1 \leqslant j \leqslant n}\left\{\left|Q Q_{1}\right|,\left|Q Q_{2}\right|,\left|Q Q_{5}\right|,\left|Q Q_{6}\right|\right\},
\end{aligned}
$$

其中点 $P_{9}\left(x_{i}, y_{j}\right)$ 和点 $Q\left(\frac{x_{i}+x_{i+1}}{2}, \frac{y_{j}+y_{j+1}}{2}\right)$ 分别为 $B_{i j}^{1}$ 和 $B_{i j}^{2}$ 的最小支集中心, 且

$$
\begin{aligned}
& \left|P_{9} P_{1}\right|=\sqrt{h_{i}^{2}+k_{j+1}^{2}}, \quad\left|P_{9} P_{7}\right|=\sqrt{h_{i+1}^{2}+k_{j+1}^{2}}, \\
& \left|P_{9} P_{5}\right|=\sqrt{h_{i+1}^{2}+k_{j}^{2}}, \quad\left|P_{9} P_{3}\right|=\sqrt{h_{i}^{2}+k_{j}^{2}} \\
& \left|Q Q_{1}\right|=\sqrt{\left(\frac{h_{i+1}}{2}\right)^{2}+\left(k_{j+2}+\frac{k_{j+1}}{2}\right)^{2}}, \\
& \left|Q Q_{2}\right|=\sqrt{\left(h_{i}+\frac{h_{i+1}}{2}\right)^{2}+\left(\frac{k_{j+1}}{2}\right)^{2}}, \\
& \left|Q Q_{5}\right|=\sqrt{\left(\frac{h_{i+1}}{2}\right)^{2}+\left(k_{j}+\frac{k_{j+1}}{2}\right)^{2}}, \\
& \left|Q Q_{6}\right|=\sqrt{\left(h_{i+2}+\frac{h_{i+1}}{2}\right)^{2}+\left(\frac{k_{j+1}}{2}\right)^{2}} .
\end{aligned}
$$


定义

$$
\begin{aligned}
h_{m n} & =\max _{-1 \leqslant i \leqslant m+2}\left\{h_{i}\right\}, \\
h_{m n} & =\max _{-1 \leqslant i \leqslant m+2}\left\{h_{i}\right\}, \\
\delta_{m n} & =\max \left\{h_{m n}, k_{m n}\right\}, \\
\delta_{m n}^{*} & =\max \left\{r_{m n, 1}, r_{m n, 2}\right\},
\end{aligned}
$$

且

$$
\begin{aligned}
& \omega^{k}(f)=\max _{l=0, \ldots, k}\left\{\omega_{\Omega}\left(\frac{\partial^{k} f}{\partial^{k-l} x \partial^{l} y}, \frac{\delta_{m n}}{2}\right)\right\}, \\
& \left\|D^{k} f\right\|=\max _{l=0, \ldots, k} \sup _{(x, y) \in \Omega}\left\{\left|\frac{\partial^{k} f}{\partial^{k-l} x \partial^{l} y}\right|\right\},
\end{aligned}
$$

其中 $k=1,2, \ldots$

记 $\|\cdot\|_{\Omega}$ 为矩形域 $\Omega$ 上的最大范数, 我们给出误差分析.

定理 4.1 设 $f(x, y) \in C(K)$, 则对充分大 $m, n \in \mathbb{Z}^{+}$,

$$
\left\|f-V_{m n}(f)\right\|_{\Omega} \leqslant \frac{7}{3} \omega_{\Omega}\left(f, \delta_{m n}^{*}\right) ;
$$

设 $f(x, y) \in C^{1}(\Omega)$, 则

$$
\left\|f-V_{m n}(f)\right\|_{\Omega} \leqslant \frac{10}{3} \delta_{m n} \omega^{1}(f)
$$

设 $f(x, y) \in C^{2}(\Omega)$, 则

$$
\left\|f-V_{m n}(f)\right\|_{\Omega} \leqslant \frac{5}{3} \delta_{m n}^{2} \omega^{2}(f)
$$

设 $f(x, y) \in C^{3}(\Omega)$, 则

$$
\left\|f-V_{m n}(f)\right\|_{\Omega} \leqslant \frac{5}{9} \delta_{m n}^{3}\left\|D^{3} f\right\| .
$$

证明 首先, 设 $f(x, y) \in C(K)$, 结合 (4.1) 和定理 3.3 , 有

$$
\begin{aligned}
\left\|f-V_{m n}(f)\right\|_{\Omega} & \leqslant \frac{|1+\lambda|}{3|1+\lambda|} \omega_{\Omega}\left(f, r_{m n, 1}\right)+\left(\frac{4}{3|\lambda|}+\frac{4}{6|\lambda|}\right)|\lambda| \omega_{\Omega}\left(f, r_{m n, 2}\right) \\
& =\frac{1}{3} \omega_{\Omega}\left(f, r_{m n, 1}\right)+2 \omega_{\Omega}\left(f, r_{m n, 2}\right) \leqslant \frac{7}{3} \omega_{\Omega}\left(f, \delta_{m n}^{*}\right) .
\end{aligned}
$$

接着, 设 $f(x, y) \in C^{1}(\Omega)$, 则由 $f(x, y)$ 于点 $\left(x^{*}, y^{*}\right)$ 的 1 阶 Taylor 展开式, 其中展开点 $(*, *)$ 可 取为 $(\cdot, y)$ 或 $(x,(\cdot)$, 即在上述三点之前分别添加相应的汉字说明. 我们得到

$$
f(x, y)=p_{1}(x, y)+\left(f_{x}^{\prime}\left(\xi_{1}, \eta_{1}\right)-f_{x}^{\prime}\left(x^{*}, y^{*}\right)\right)\left(x-x^{*}\right)+\left(f_{y}^{\prime}\left(\xi_{1}, \eta_{1}\right)-f_{y}^{\prime}\left(x^{*}, y^{*}\right)\right)\left(y-y^{*}\right),
$$

其中

$$
p_{1}(x, y)=f\left(x^{*}, y^{*}\right)+f_{x}^{\prime}\left(x^{*}, y^{*}\right)\left(x-x^{*}\right)+f_{y}^{\prime}\left(x^{*}, y^{*}\right)\left(y-y^{*}\right),
$$

且 $\left(\xi_{1}, \eta_{1}\right)=\tau_{1}(x, y)+\left(1-\tau_{1}\right)\left(x^{*}, y^{*}\right), \tau_{1} \in(0,1)$.

因此, 由 (4.6) 和定理 3.3 , 成立

$$
\left\|f-V_{m n}(f)\right\|_{\Omega}=\left\|f-V_{m n}(f)\right\|_{\Omega} \leqslant\left\|f-p_{1}\right\|_{\Omega}+\left\|V_{m n}\left(f-p_{1}\right)\right\|_{\Omega}
$$




$$
\begin{aligned}
& \leqslant\left\|f-p_{1}\right\|_{\Omega}+\left\|V_{m n}\right\|_{\Omega} \cdot\left\|f-p_{1}\right\|_{\Omega} \leqslant \frac{10}{3}\left\|f-p_{1}\right\|_{\Omega} \\
& \leqslant \frac{10}{3}\left(\frac{h_{m n}}{2} \omega_{\Omega}\left(f_{x}^{\prime}, \frac{\delta_{m n}}{2}\right)+\frac{k_{m n}}{2}+\omega_{\Omega}\left(f_{y}^{\prime}, \frac{\delta_{m n}}{2}\right)\right) \leqslant \frac{10}{3} \delta_{m n} \omega^{1} f,
\end{aligned}
$$

其中 $\left\|V_{m n}\right\|_{\Omega}=\frac{|1+\lambda|}{3|1+\lambda|}+\left(\frac{4}{3|\lambda|}+\frac{4}{6|\lambda|}\right)|\lambda|=\frac{7}{3}$.

然后, 设 $f(x, y) \in C^{2}(\Omega)$, 则由 $f(x, y)$ 于点 $\left(x^{*}, y^{*}\right)$ 的 2 阶 Taylor 展开式, 其中展开点 $(*, *)$ 可 取为 $(\cdot, y)$ 或 $(x, \cdot)$, 即在上述三点之前分别添加相应的汉字说明, 有

$$
\begin{aligned}
f(x, y)= & p_{2}(x, y)+\frac{1}{2}\left\{\left[f_{x x}^{\prime \prime}\left(\xi_{2}, \eta_{2}\right)-f_{x x}^{\prime \prime}\left(x^{*}, y^{*}\right)\right]\left(x-x^{*}\right)^{2}+2\left[f_{x y}^{\prime \prime}\left(\xi_{2}, \eta_{2}\right)-f_{x y}^{\prime \prime}\left(x^{*}, y^{*}\right)\right]\right. \\
& \left.\times\left(x-x^{*}\right)\left(y-y^{*}\right)+\left[f_{y y}^{\prime \prime}\left(\xi_{2}, \eta_{2}\right)-f_{y y}^{\prime \prime}\left(x^{*}, y^{*}\right)\right]\left(y-y^{*}\right)^{2}\right\},
\end{aligned}
$$

其中

$$
p_{2}(x, y)=p_{1}(x, y)+\frac{1}{2}\left[\left(x-x^{*}\right) \frac{\partial}{\partial x}+\left(y-y^{*}\right) \frac{\partial}{\partial y}\right]^{2}\left(x^{*}, y^{*}\right),
$$

且 $\left(\xi_{2}, \eta_{2}\right)=\tau_{2}(x, y)+\left(1-\tau_{2}\right)\left(x^{*}, y^{*}\right), \tau_{2} \in(0,1)$.

故而, 由 (4.6)、定理 3.3 和 $\left\|V_{m n}\right\|_{\Omega}=\frac{7}{3}$, 成立

$$
\begin{aligned}
\left\|f-V_{m n}(f)\right\|_{\Omega} & \leqslant\left\|f-V_{m n}(f)\right\|_{\Omega} \leqslant\left\|f-p_{2}\right\|_{\Omega}+\left\|V_{m n}\left(f-p_{2}\right)\right\|_{\Omega} \leqslant \frac{10}{3}\left\|f-p_{2}\right\|_{\Omega} \\
& \leqslant \frac{10}{3} \times \frac{1}{2}\left[\frac{h_{m n}^{2}}{4} \omega_{\Omega}\left(f_{x x}^{\prime \prime}, \frac{\delta_{m n}}{2}\right)+\frac{h_{m n} k_{m n}}{2} \omega_{\Omega}\left(f_{x y}^{\prime \prime}, \frac{\delta_{m n}}{2}\right)+\frac{k_{m n}^{2}}{4} \omega_{\Omega}\left(f_{y y}^{\prime \prime}, \frac{\delta_{m n}}{2}\right)\right] \\
& \leqslant \frac{10}{3} \times \frac{1}{2} \delta_{m n}^{2} \omega^{2} f=\frac{5}{3} \delta_{m n}^{2} \omega^{2} f .
\end{aligned}
$$

最后, 设 $f(x, y) \in C^{3}(\Omega)$, 则借助 $f(x, y)$ 于点 $\left(x^{*}, y^{*}\right)$ 的 3 阶 Taylor 展开式, 成立 $f(x, y)=p_{2}(x, y)$ $+\frac{1}{3 !}\left[\left(x-x^{*}\right) \frac{\partial}{\partial x}+\left(y-y^{*}\right) \frac{\partial}{\partial y}\right]^{3} f\left(\xi_{3}, \eta_{3}\right)$, 其中 $\left(\xi_{3}, \eta_{3}\right)=\tau_{3}(x, y)+\left(1-\tau_{3}\right)\left(x^{*}, y^{*}\right), \tau_{3} \in(0,1)$.

因此, 由 (4.7), 我们得到此时的一个误差上界 $\left\|f-V_{m n}(f)\right\|_{\Omega} \leqslant \frac{10}{3}\left\|f-p_{2}\right\|_{\Omega} \leqslant \frac{10}{3} \times \frac{1}{6}\left(\frac{h_{m n}}{2}+\frac{k_{m n}}{2}\right)^{3}$ $\times\left\|D^{3} f\right\| \leqslant \frac{5}{9} \delta_{m n}^{3}\left\|D^{3} f\right\|$.

综上所述, 我们证明了结论 (4.8)-(4.11) 成立.

注 3 设 $f \in C^{r}(\Omega), r=0,1,2$, 当 $\delta_{m n} \rightarrow 0^{+}$时, $V_{m n}(f)$ 一致逼近于 $f$.

致谢 作者非常感谢李崇君教授曾提出的宝贵见解，以及审稿人所提出的诸多宝贵建议，这些建议有益于提高论文质 量与可读性.

\section{参考文献}

1 Farin G. Curves and Surfaces for Computer Aided Geometric Design: A Practical Guide. Boston: Academic Press, 1992

2 Piegl L, Tiller W. The NURBS Book. Berlin: Spring-Verlag, 1997

3 Schumaker L L. Spline Functions: Basic Theory. Malabar FL: Krieger Publishing Company, 1993

4 王国瑾, 汪国昭, 郑建民. 计算机辅助几何设计. 北京: 高等教育出版社, 2001

5 Zhang J W. C-curves: An extension of cubic curves. Comput Aided Geom Design, 1996, 13: 119-127

6 Zhang J W. C-Bézier curves and surfaces. Graphs Models Image Process, 1999, 61: 2-15

7 Chen Q Y, Wang G Z. A class of Bézier-like curves. Comput Aided Geom Design, 2003, 20: 29-39

8 Wang G Z, Chen Q Y, Zhou M H. NUAT B-spline curves. Comput Aided Geom Design, 2004, 21: 193-205

9 钱江, 唐月红. H Bézier-Like 曲线在工程中的应用. 数值计算与计算机应用, 2007, 28: 167-178

10 钱江, 刘华勇. UAH B 样条与均匀 B 样条关系研究. 河海大学学报, 2006, 34: 599-602 
11 Qian J, Tang Y H. On non-uniform Algebraic-Hyperbolic (NUAH) B-splines. Numer Math J Chin Univ, 2006, 4: 320-335

12 王仁宏. 多元齿的结构与插值. 数学学报, 1975, 18: 91-106

13 Wang R H. Multivariate Spline Functions and Their Applications. Beijing: Science Press, 2001

$14 \mathrm{Li} \mathrm{C} \mathrm{J,} \mathrm{Chen} \mathrm{J.} \mathrm{On} \mathrm{the} \mathrm{dimensions} \mathrm{of} \mathrm{bivariate} \mathrm{spline} \mathrm{spaces} \mathrm{and} \mathrm{their} \mathrm{stability.} \mathrm{J} \mathrm{Comput} \mathrm{Appl} \mathrm{Math,} \mathrm{2011,} \mathrm{236:}$ $765-774$

$15 \mathrm{Xu} \mathrm{Z} \mathrm{Q}$, Wang R H. The instability degree in the dimension of spaces of bivariate spline. Approx Theory Appl, 2002, 18: $68-80$

16 Shi X Q. The singularity of Morgan-Scott triangulation. Comput Aided Geom Design, 1991, 8: 201-206

17 Li C J, Wang R H. Bivariate cubic spline space and bivariate cubic NURBS surfaces. In: Proceedings of Geometric Modeling and Processing. New Jersey: IEEE Computer Society Press, 2004, 115-123

18 Wang R H, Li C J. Bivariate quartic spline spaces and quasi-interpolation operators. J Comput Appl Math, 2006, 190: 325-338

19 Wang S M. Spline interpolation over type-2 triangulations. Appl Math Comput, 1992, 49: 299-313

20 Qian J, Wang R H, Li C J. The bases of non-uniform cubic spline space $S_{3}^{1,2}\left(\Delta_{m n}^{(2)}\right)$. Numer Math Theory Methods Appl, 2012, 5: 635-652

21 Lai Y S, Du W P, Wang R H. The Viro method for construction of Bernstein-Bézier algebraic hypersurface piece. Sci China Math, 2012, 55: 1269-1279

22 赖义生, 王仁宏. 参系数零维分片代数簇的实零点. 中国科学 A 辑, 2008, 38: 1153-1167

23 朱春钢, 王仁宏. 拟贯穿剖分上分片代数曲线的 Nöther 型定理. 中国科学 A 辑, 2009, 39: 27-33

24 Lai Y S, Wang R H, Wu J M. Solving parametric piecewise polynomial systems. J Comput Appl Math, 2011, 236: 924-936

25 朱春钢, 王仁宏. 三角剖分上分片代数曲线的 Nöther 型定理. 中国科学 A 辑, 2007, 37: 425-430

26 Deng J S, Feng Y Y, Kozak J. A note on the dimension of the bivariate spline space over the Morgan-Scott triangulation. SIAM J Numer Anal, 2000, 37: 1021-1028

27 Wang R H, Lu Y. Quasi-interpolating operators and their applications in hypersingular integrals. J Comput Math, 1998, 16: 337-344

28 Wang R H, Lu Y. Quasi-interpolating operators in $S_{2}^{1}\left(\Delta_{m n}^{2} *\right)$ on non-uniform type-2 triangulations. Numer Math J Chin Univ (Engl Ser), 1999, 2: 97-103

29 Dagnino C, Lamberti P. On the approximation power of bivariate quadratic $C^{1}$ splines. J Comput Appl Math, 2001, 131: $321-332$

30 Dagnino C, Lamberti P. Some performances of local bivariate quadratic $C^{1}$ quasi-interpolating splines on nonuniform type-2 triangulations. J Comput Appl Math, 2005, 173: 21-37

31 Qian J, Wang F. On the approximation of the derivatives of spline quasi-interpolation in cubic spline space $S_{3}^{1,2}\left(\Delta_{m n}^{(2)}\right)$. Numer Math Theory Methods Appl, 2014, 7: 1-22

\section{On spline quasi-interpolation in cubic spline space $S_{3}^{1,2}\left(\Delta_{m n}^{(2)}\right)$}

\section{QIAN Jiang, WANG RenHong, ZHU ChunGang \& WANG Fan}

Abstract In this paper, by means of the basis composed of two sets of splines with distinct local supports, cubic spline quasi-interpolating operators are investigated on nonuniform type-2 triangulation. These variation diminishing operators based on five mesh points or the center of the support of each spline $B_{i j}^{1}$ and five mesh points of the support of each spline $B_{i j}^{2}$ can preserve good approximation, and even reproduce any polynomial of nearly best degrees. Moreover, the spline series can approximate a real sufficiently smooth function uniformly based on the modulus of continuity. And then the convergence results are worked out.

Keywords bivariate splines, conformality of smoothing cofactor method, nonuniform type-2 triangulation, quasi-interpolation, modulus of continuity

$\operatorname{MSC}(2010) \quad 65 \mathrm{D} 07,41 \mathrm{A25}$

doi: 10.1360/N012013-00140 